\title{
ON THE RATE OF CONVERGENCE OF DIFFERENCE SCHEMES FOR THE POISSON EQUATION WITH DYNAMIC INTERFACE CONDITIONS
}

\author{
BOŠKO S. JOVANOVIĆ \\ University of Belgrade, Faculty of Mathematics \\ Studentski trg 16, 11000 Belgrade, Serbia and Montenegro \\ E-mail: bosko@matf.bg.ac.yu \\ LUBIN G. VULKOV \\ Department of Mathematics, University of Rousse \\ Studentska str. 8, 7017 Rousse, Bulgaria \\ E-mail: vulkov@ami.ru.acad.bg
}

Dedicated to Raytcho Lazarov on the occasion of his 60th birthday.

\begin{abstract}
The convergence of difference schemes for the two-dimensional weakly parabolic equation (elliptic equation with a dynamic interface condition) is studied. Estimates for the rate of convergence "almost" (except for the logarithmic factor) compatible with the smoothness of the differential problem solution in special discrete Sobolev norms are obtained.
\end{abstract}

2000 Mathematics Subject Classification: 65M15.

Keywords: weakly parabolic equation (elliptic equation with a dynamic interface condition), difference scheme, weak solution, rate of convergence.

\section{Introduction}

The modern theory of convergence of difference schemes for elliptic partial differential equations was started by A. A. Samarskii, R. D. Lazarov and V. L. Makarov [20]. According to [15], estimates for elliptic problems of the type

$$
\|u-v\|_{W_{p}^{k}(\omega)} \leqslant C h^{s-k}\|u\|_{W_{p}^{s}(\Omega)}, \quad k<s
$$

are called estimates compatible with the smoothness of the solution $u$. Here $v$ is a discrete solution, $h$ is a discretization parameter, and $W_{p}^{k}(\omega)$ is a discrete Sobolev space. Such estimates can not be derived by the Taylor formula because the functions belong to Sobolev spaces and the Bramble-Hilbert lemma and its generalizations [2,5] are often used.

The ideas of [20] have been further developed in [7] to obtain convergence rate estimates for parabolic and hyperbolic problems. 
Interface problems model many phenomena in science and engineering and various forms of jump (interface) relations satisfied by the solution and its derivatives are known $[6,16]$. In [3], a review of the recent results on numerical solutions of elliptic and parabolic twodimensional interface problems is presented. In $[11,13,23]$, the convergence of FDM for different elliptic, parabolic, and hyperbolic problems in which the solution is continuous on the interface curves, while the flow is discontinuous, has been studied.

In the present work, a weakly parabolic problem is considered i.e, a problem in which the solution satisfies an elliptic equation in two adjacent regions, and on the interface - a dynamic conjugation condition. The existence of a generalized solution for different assumptions of smoothness of the input data is proved in the next section. For the numerical solution the implicit difference scheme with an averaged right-hand side is analyzed in Section 3. The convergence of this scheme in special discrete Sobolev norms is proved in Section 4. Similar results for weighted difference schemes also hold.

\section{Regularity of the continuous solution}

Let $\Omega=\left(0, l_{1}\right) \times\left(0, l_{2}\right), \Gamma=\partial \Omega, x_{2}^{0} \in\left(0, l_{2}\right), S=\left(0, l_{1}\right) \times\left\{x_{2}^{0}\right\}, \Omega^{-}=\left(0, l_{1}\right) \times\left(0, x_{2}^{0}\right)$ and $\Omega^{+}=\left(0, l_{1}\right) \times\left(x_{2}^{0}, l_{2}\right)$. We denote $Q=\Omega \times(0, T), Q^{-}=\Omega^{-} \times(0, T)$ and $Q^{+}=\Omega^{+} \times(0, T)$. We consider the initial boundary-value problem (IBVP) with the dynamic boundary condition

$$
\begin{aligned}
-\Delta u & =f(x, t), \quad x=\left(x_{1}, x_{2}\right) \in \Omega^{-} \cup \Omega^{+}, \quad t \in(0, T), \\
{[u]_{S}=0, \quad \frac{\partial u}{\partial t} } & =\left[\frac{\partial u}{\partial \nu}\right]_{S}+g(x, t), \quad x \in S, \quad t \in(0, T), \\
u(x, t) & =0, \quad x \in \Gamma, \quad t \in(0, T), \\
u(x, 0) & =u_{0}(x), \quad x \in S,
\end{aligned}
$$

where $\Delta=\partial^{2} / \partial x_{1}^{2}+\partial^{2} / \partial x_{2}^{2}$ is the Laplace operator, $\partial / \partial \nu=\partial / \partial x_{2}$ is the normal derivative on $S$ and $[u]_{S}=u\left(x_{1}, x_{2}^{0}+0, t\right)-u\left(x_{1}, x_{2}^{0}-0, t\right)$. A similar problem is considered in [21].

In a standard manner one obtains a weak form of IBVP (1)

$$
\iint_{0}^{T} \frac{\partial u}{\partial t} v d S d t+\int_{0}^{T} \int_{\Omega} \nabla u \cdot \nabla v d x d t=\int_{0}^{T} \int_{\Omega} f v d x d t+\iint_{0}^{T} g v d S d t \quad \forall v \in \stackrel{\circ}{W_{S}^{1,0}}(Q),
$$

where $\nabla u \cdot \nabla v=\frac{\partial u}{\partial x_{1}} \frac{\partial v}{\partial x_{1}}+\frac{\partial u}{\partial x_{2}} \frac{\partial v}{\partial x_{2}}$ and $\stackrel{\circ}{W_{2}^{1,0}}(Q)=\left\{v \in W_{2}^{1,0}(Q): v=0\right.$ on $\left.\Gamma \times(0, T)\right\}$.

The same weak form corresponds to the following IBVP:

$$
\begin{aligned}
\delta_{S} \frac{\partial u}{\partial t}-\Delta u & =\varphi(x, t), & (x, t) & \in Q, \\
u(x, t) & =0, & (x, t) & \in \Gamma \times(0, T), \\
u(x, 0) & =u_{0}(x), & x & \in S,
\end{aligned}
$$

where $\delta_{S}(x)=\delta\left(x_{2}-x_{2}^{0}\right)$ is the Dirac delta function concentrated on $S, \varphi(x, t)=f(x, t)+$ $\delta_{S}(x) g(x, t)$, where the equality is considered in the sense of the theory of distributions [22]. In this sense, IBVPs (1) and (2) are equivalent. Further, problem (2) will be called a weakly parabolic problem since its parabolic nature appears only on $S \times(0, T)$. 
The following abstract Cauchy problem correspond to the IBVP (2):

$$
B u^{\prime}+A u=\varphi(t), \quad t \in(0, T) ; \quad B u(0)=B u_{0}
$$

where $u_{0}$ is a given element of the Hilbert space $H, \varphi(t)$ is given and $u(t)$ is the unknown function with values in $H, A$ is unbounded a self-adjoint positively definite linear operator in $H$, and $B$ is a self-adjoint nonnegative linear operator in $H$. We assume that the domain $D(A)$ of the operator $A$ is dense in $H$ and $D(A) \subset D(B) \subset H$. Also the operator inequality $A \geqslant B$ is supposed. The inner product and the norm in $H$ are denoted by $(u, v)$ and $\|u\|$, respectively. Problems of this type have been extensively investigated (see e.g. $[4,18,24]$ and the references therein).

In a standard fashion we introduce the inner products $(u, v)_{A}=(A u, v),(u, v)_{A^{-1}}=$ $\left(A^{-1} u, v\right)$ and $(u, v)_{B}=(B u, v)$ and the corresponding energy spaces $H_{A}, H_{A^{-1}}$ and $H_{B}$. We also introduce the Sobolev space $W_{2}^{k}(0, T ; H)$ of functions which map $(0, T)$ into $H$ [17]. Specially, by $\widetilde{W}_{2}^{1 / 2}(0, T ; H)$ we denote the space of functions $u:(0, T) \rightarrow H$ with the norm

$$
\|u\|_{\widetilde{W}_{2}^{1 / 2}(0, T ; H)}^{2}=\int_{0}^{T} \int_{0}^{T} \frac{\left\|u(t)-u\left(t^{\prime}\right)\right\|^{2}}{\left|t-t^{\prime}\right|^{2}} d t d t^{\prime}+\int_{0}^{T}\left(\frac{1}{t}+\frac{1}{T-t}\right)\|u(t)\|^{2} d t .
$$

The following assertion holds [12]:

Lemma 1. For the solution of (3) the following a priori estimates hold:

$$
\int_{0}^{T}\|u(t)\|_{A}^{2} d t+\int_{0}^{T} \int_{0}^{T} \frac{\left\|u(t)-u\left(t^{\prime}\right)\right\|_{B}^{2}}{\left|t-t^{\prime}\right|^{2}} d t d t^{\prime}+\max _{t \in[0, T]}\|u(t)\|_{B}^{2} \leqslant C\left(\left\|u_{0}\right\|_{B}^{2}+\int_{0}^{T}\|\varphi(t)\|_{A^{-1}}^{2} d t\right)
$$

and

$$
\int_{0}^{T}\|u(t)\|_{B}^{2} d t+\max _{t \in[0, T]}\|B u(t)\|_{A^{-1}}^{2} \leqslant C\left(\left\|B u_{0}\right\|_{A^{-1}}^{2}+\int_{0}^{T}\left\|A^{-1} \varphi(t)\right\|_{B}^{2} d t\right) .
$$

In the case where $\varphi(t)=B \psi^{\prime}(t)$, we have

$$
\begin{aligned}
\int_{0}^{T}\|u(t)\|_{A}^{2} d t & +\int_{0}^{T} \int_{0}^{T} \frac{\left\|u(t)-u\left(t^{\prime}\right)\right\|_{B}^{2}}{\left|t-t^{\prime}\right|^{2}} d t d t^{\prime}+\max _{t \in[0, T]}\|u(t)\|_{B}^{2} \\
& \leqslant C\left\{\left\|u_{0}\right\|_{B}^{2}+\int_{0}^{T} \int_{0}^{T} \frac{\left\|\psi(t)-\psi\left(t^{\prime}\right)\right\|_{B}^{2}}{\left|t-t^{\prime}\right|^{2}} d t d t^{\prime}+\int_{0}^{T}\left(\frac{1}{t}+\frac{1}{T-t}\right)\|\psi(t)\|_{B}^{2} d t\right\}
\end{aligned}
$$

and

$$
\int_{0}^{T}\|u(t)\|_{B}^{2} d t+\max _{t \in[0, T]}\|B u(t)\|_{A^{-1}}^{2} \leqslant C\left(\left\|B u_{0}\right\|_{A^{-1}}^{2}+\max _{t \in[0, T]}\|B \psi(t)\|_{A^{-1}}^{2}+\int_{0}^{T}\|\psi(t)\|_{B}^{2} d t\right) .
$$


In problem (2) we have: $H=L_{2}(\Omega), A u=-\Delta u, B u=\delta_{S} u, H_{A}=\stackrel{\circ}{W_{2}^{1}}(\Omega), H_{B}=L_{2}(S)$ and

$$
\begin{aligned}
\|u\|_{A}^{2} & =\int_{\Omega}\left[\left(\frac{\partial u}{\partial x_{1}}\right)^{2}+\left(\frac{\partial u}{\partial x_{2}}\right)^{2}\right] d x \asymp\|u\|_{W_{2}^{1}(\Omega)}^{2}, \quad u \in \stackrel{\circ}{W_{2}^{1}(\Omega),} \\
\|u\|_{B}^{2} & =\int_{S} u^{2} d S=\|u\|_{L_{2}(S)}^{2} .
\end{aligned}
$$

Therefore, $H_{A^{-1}}=W_{2}^{-1}(\Omega)$ and $\|u\|_{A^{-1}} \asymp\|u\|_{W_{2}^{-1}(\Omega)}$. By $\widetilde{W}_{2}^{1 / 2}(S)$ we denote the space of functions $u\left(x_{1}, x_{2}^{0}\right)$ defined on $S$, with the norm

$$
\|u\|_{\widetilde{W}_{2}^{1 / 2}(S)}^{2}=\int_{0}^{l_{1}} \int_{0}^{l_{1}}\left[\frac{u\left(x_{1}, x_{2}^{0}\right)-u\left(x_{1}^{\prime}, x_{2}^{0}\right)}{x_{1}-x_{1}^{\prime}}\right]^{2} d x_{1} d x_{1}^{\prime}+\int_{0}^{l_{1}}\left(\frac{1}{x_{1}}+\frac{1}{l_{1}-x_{1}}\right) u^{2}\left(x_{1}, x_{2}^{0}\right) d x_{1}
$$

and by $\widetilde{W}_{2}^{-1 / 2}(S)$ - its dual space. As is well known, the inner product $(u, v)_{L_{2}(S)}$ can be continuously extended to the map $(u, v)_{\widetilde{W}_{2}^{-1 / 2}(S) \times \widetilde{W}_{2}^{1 / 2}(S)}$ from $\widetilde{W}_{2}^{-1 / 2}(S) \times \widetilde{W}_{2}^{1 / 2}(S)$ into $R$, and the norm in $\widetilde{W}_{2}^{-1 / 2}(S)$ is defined as follows:

$$
\|u\|_{\widetilde{W}_{2}^{-1 / 2}(S)}=\sup _{v \in \widetilde{W}_{2}^{1 / 2}(S)} \frac{\left|(u, v)_{\widetilde{W}_{2}^{-1 / 2}(S) \times \widetilde{W}_{2}^{1 / 2}(S)}\right|}{\|v\|_{\widetilde{W}_{2}^{1 / 2}(S)}} .
$$

From the results of [1] it follows that

$$
\|u\|_{\widetilde{W}_{2}^{1 / 2}(S)} \asymp \inf _{\substack{v W_{2}^{1}(\Omega),\left.v\right|_{S}=u}}\|v\|_{W_{2}^{1}(\Omega)} .
$$

Also, it is easy to prove that

$$
\|B u\|_{A^{-1}} \asymp\|u\|_{\widetilde{W}_{2}^{-1 / 2}(S)} .
$$

In this way, the a priori estimates (4) and (5) in the case of the IBVP (2), take the form

$$
\begin{aligned}
\int_{0}^{T}\|u(\cdot, t)\|_{W_{2}^{1}(\Omega)}^{2} d t & +\int_{0}^{T} \int_{0}^{T} \frac{\left\|u(\cdot, t)-u\left(\cdot, t^{\prime}\right)\right\|_{L_{2}(S)}^{2} d t d t^{\prime}+\max _{t \in[0, T]}\|u(\cdot, t)\|_{L_{2}(S)}^{2}}{\left|t-t^{\prime}\right|^{2}} \\
& \leqslant C\left(\left\|u_{0}\right\|_{L_{2}(S)}^{2}+\int_{0}^{T}\|f(\cdot, t)\|_{W_{2}^{-1}(\Omega)}^{2} d t+\int_{0}^{T}\|g(\cdot, t)\|_{\widetilde{W}_{2}^{-1 / 2}(S)}^{2} d t\right)
\end{aligned}
$$

and

$$
\int_{0}^{T}\|u(\cdot, t)\|_{L_{2}(S)}^{2} d t+\max _{t \in[0, T]}\|u(\cdot, t)\|_{\widetilde{W}_{2}^{-1 / 2}(S)}^{2} \leqslant C\left(\left\|u_{0}\right\|_{\widetilde{W}_{2}^{-1 / 2}(S)}^{2}+\int_{0}^{T}\left\|A^{-1} \varphi(\cdot, t)\right\|_{L_{2}(S)} d t\right) .
$$

Remark 1. The proof of Lemma 1 can be found in [8] (for $B=I=$ identity operator) and [12] (for $B>0$ ). The Fourier expansion is used to obtain estimates (4) and (6). Estimates of 
these types for the parabolic PDEs obtained using the Fourier transform are given in [14]. In our case, the operator $B$ is only non-negative and (4) leads to the nonstandard a priory estimate (7). Note that the functional space containing the generalized solution of the IBVP (1) is determinated by (7): if $u_{0} \in L_{2}(S), f \in L_{2}\left(0, T ; W_{2}^{-1}(\Omega)\right)$ and $g \in L_{2}\left(0, T ; \widetilde{W}_{2}^{-1 / 2}(S)\right)$, then $u \in L_{2}\left(0, T ; W_{2}^{1}(\Omega)\right) \cap W_{2}^{1 / 2}\left(0, T ; L_{2}(S)\right)$.

Using Lemma 1 and equation (1), we get the following assertion:

Lemma 2. If $f \in L_{2}(Q), g \in L_{2}\left(0, T ; \widetilde{W}_{2}^{1 / 2}(S)\right)$ and $u_{0} \in \stackrel{\circ}{W_{2}^{1}}(S)$, then the solution of the IBVP (2) belongs to the space $L_{2}\left(0, T ; W_{2}^{2}\left(\Omega^{-}\right)\right) \cap L_{2}\left(0, T ; W_{2}^{2}\left(\Omega^{+}\right)\right) \cap W_{2}^{1}\left(0, T ; L_{2}(S)\right)$ and the following a priori estimate is fulfilled:

$$
\begin{aligned}
\int_{0}^{T}\left(\left\|\frac{\partial^{2} u}{\partial x_{1}^{2}}(\cdot, t)\right\|_{L_{2}(\Omega)}^{2}\right. & +\left\|\frac{\partial^{2} u}{\partial x_{1} \partial x_{2}}(\cdot, t)\right\|_{L_{2}(\Omega)}^{2}+\left\|\frac{\partial^{2} u}{\partial x_{2}^{2}}(\cdot, t)\right\|_{L_{2}\left(\Omega^{-}\right)}^{2} \\
& \left.+\left\|\frac{\partial^{2} u}{\partial x_{2}^{2}}(\cdot, t)\right\|_{L_{2}\left(\Omega^{+}\right)}^{2}+\left\|\frac{\partial u}{\partial t}(\cdot, t)\right\|_{L_{2}(S)}^{2}\right) d t+\max _{t \in[0, T]}\|u(\cdot, t)\|_{W_{2}^{1}(S)}^{2} \\
& \leqslant C\left(\left\|u_{0}\right\|_{W_{2}^{1}(S)}^{2}+\int_{0}^{T}\|f(\cdot, t)\|_{L_{2}(\Omega)}^{2} d t+\int_{0}^{T}\|g(\cdot, t)\|_{\widetilde{W}_{2}^{1 / 2}(S)}^{2} d t\right) .
\end{aligned}
$$

If $f \in L_{2}\left(0, T ; W_{2}^{1}(\Omega)\right), f=0$ at $x_{1}=0$ and $x_{1}=l_{1}, g \in L_{2}\left(0, T ; W_{2}^{3 / 2}(S)\right)$ and $u_{0} \in$ $W_{2}^{2}(S) \cap W_{2}^{1}(S)$, then the solution of the IBVP (1) belongs to the space $L_{2}\left(0, T ; W_{2}^{3}\left(\Omega^{-}\right)\right) \cap$ $\left.L_{2}\left(0, T ; W_{2}^{3}\left(\Omega^{+}\right)\right), \frac{\partial u}{\partial x_{1}} \in W_{2}^{1}\left(0, T ; L_{(} S\right)\right) \cap W_{2}^{1 / 2}\left(0, T ; W_{2}^{1}(S)\right)$ and the following estimate holds:

$$
\begin{aligned}
\int_{0}^{T}\left(\left\|\frac{\partial^{3} u}{\partial x_{1}^{3}}(\cdot, t)\right\|_{L_{2}(\Omega)}^{2}\right. & +\left\|\frac{\partial^{3} u}{\partial x_{1}^{2} \partial x_{2}}(\cdot, t)\right\|_{L_{2}(\Omega)}^{2}+\left\|\frac{\partial^{3} u}{\partial x_{1} \partial x_{2}^{2}}(\cdot, t)\right\|_{L_{2}\left(\Omega^{-}\right)}^{2}+\left\|\frac{\partial^{3} u}{\partial x_{1} \partial x_{2}^{2}}(\cdot, t)\right\|_{L_{2}\left(\Omega^{+}\right)}^{2} \\
& \left.+\left\|\frac{\partial^{3} u}{\partial x_{2}^{3}}(\cdot, t)\right\|_{L_{2}\left(\Omega^{-}\right)}^{2}+\left\|\frac{\partial^{3} u}{\partial x_{2}^{3}}(\cdot, t)\right\|_{L_{2}\left(\Omega^{+}\right)}^{2}+\left\|\frac{\partial^{2} u}{\partial t \partial x_{1}}(\cdot, t)\right\|_{L_{2}(S)}^{2}\right) d t \\
& +\int_{0}^{T} \frac{\left\|\frac{\partial^{2} u}{\partial x_{1}^{2}}(\cdot, t)-\frac{\partial^{2} u}{\partial x_{1}^{2}}\left(\cdot, t^{\prime}\right)\right\|_{L_{2}(S)}^{2}}{\left|t-t^{\prime}\right|^{2}} d t d t^{\prime}+\max _{t \in[0, T]}\|u(\cdot, t)\|_{W_{2}^{2}(S)}^{2} \\
& \leqslant C\left(\left\|u_{0}\right\|_{W_{2}^{2}(S)}^{2}+\int_{0}^{T}\|f(\cdot, t)\|_{W_{2}^{1}(\Omega)}^{2} d t+\int_{0}^{T}\|g(\cdot, t)\|_{W_{2}^{3 / 2}(S)}^{2} d t\right) .
\end{aligned}
$$

If, in addition, $\frac{\partial f}{\partial t} \in L_{2}\left(0, T ; W_{2}^{-1}(\Omega)\right), f(\cdot, 0) \in L_{2}(\Omega), g \in \widetilde{W}_{2}^{1 / 2}\left(0, T ; L_{2}(S)\right)$ and $g(\cdot, 0)=$ 
0 , then $\frac{\partial u}{\partial t} \in L_{2}\left(0, T ; W_{2}^{1}(\Omega)\right) \cap W_{2}^{1 / 2}\left(0, T ; L_{2}(S)\right)$ and the following estimate holds:

$$
\begin{aligned}
& \int_{0}^{T}\left(\left\|\frac{\partial^{2} u}{\partial t \partial x_{1}}(\cdot, t)\right\|_{L_{2}(\Omega)}^{2}+\left\|\frac{\partial^{2} u}{\partial t \partial x_{2}}(\cdot, t)\right\|_{L_{2}(\Omega)}^{2}\right) d t+\int_{0}^{T} \frac{\left\|\frac{\partial u}{\partial t}(\cdot, t)-\frac{\partial u}{\partial t}\left(\cdot, t^{\prime}\right)\right\|_{L_{2}(S)}^{2}}{\left|t-t^{\prime}\right|^{2}} d t d t^{\prime} \\
& +\max _{t \in[0, T]}\left\|\frac{\partial u}{\partial t}(\cdot, t)\right\|_{L_{2}(S)}^{2} \leqslant C\left[\left\|u_{0}\right\|_{W_{2}^{2}(S)}^{2}+\|f(\cdot, 0)\|_{L_{2}(\Omega)}^{2}+\int_{0}^{T}\left\|\frac{\partial f}{\partial t}(\cdot, t)\right\|_{W_{2}^{-1}(\Omega)}^{2} d t\right. \\
& \left.+\int_{0}^{T} \int_{0}^{T} \frac{\left\|g(\cdot, t)-g\left(\cdot, t^{\prime}\right)\right\|_{L_{2}(S)}^{2}}{\left|t-t^{\prime}\right|^{2}} d t d t^{\prime}+\int_{0}^{T}\left(\frac{1}{t}+\frac{1}{T-t}\right)\|g(\cdot, t)\|_{L_{2}(S)}^{2} d t\right] .
\end{aligned}
$$

\section{Difference approximation}

Let $\omega_{i}$ be a uniform mesh with a step $h_{i}=l_{i} / N_{i}$ in $\left(0, l_{i}\right), i=1,2$. For simplicity, we suppose that $x_{2}^{0}$ is a mesh point of $\omega_{2}$. Suppose that $\omega_{i}^{-}=\omega_{i} \cup\{0\}, \bar{\omega}_{i}=\omega_{i} \cup\left\{0, l_{i}\right\}, \omega_{h}=\omega_{1} \times \omega_{2}$, $\bar{\omega}_{h}=\bar{\omega}_{1} \times \bar{\omega}_{2}, \gamma_{h}=\bar{\omega}_{h} \cap \Gamma, \sigma_{h}=\omega_{h} \cap S$ and $\sigma_{h}^{-}=\sigma_{h} \cup\left\{\left(0, x_{2}^{0}\right)\right\}$. Let $\omega_{\tau}$ be a uniform mesh with a step $\tau=T / N$ in $(0, T), \omega_{\tau}^{+}=\omega_{\tau} \cup\{T\}$ and $\bar{\omega}_{\tau}=\omega_{\tau} \cup\{0, T\}$.

Further, we shall use the standard notations [19]

$$
\begin{aligned}
v_{x_{i}}=\frac{v\left(x+h_{i} e_{i}, t\right)-v(x, t)}{h_{i}}, & v_{\bar{x}_{i}}=\frac{v(x, t)-v\left(x-h_{i} e_{i}, t\right)}{h_{i}}, \quad e_{1}=(1,0), \quad e_{2}=(0,1), \\
v_{t}=\frac{v(x, t+\tau)-v(x, t)}{\tau}, & v_{\bar{t}}=\frac{v(x, t)-v(x, t-\tau)}{\tau} .
\end{aligned}
$$

We shall approximate the $\operatorname{IBVP}(2)$ on the mesh $\bar{Q}_{h \tau}=\bar{\omega}_{1} \times \bar{\omega}_{2} \times \bar{\omega}_{\tau}$ by the implicit difference scheme with the averaged right-hand side

$$
\begin{aligned}
\delta_{\sigma_{h}} v_{\bar{t}}-\Delta_{h} v & =T_{1}^{2} T_{2}^{2} T_{t}^{-} \varphi, & (x, t) & \in \omega_{h} \times \omega_{\tau}^{+}, \\
v(x, t) & =0, & (x, t) & \in \gamma_{h} \times \omega_{\tau}^{+}, \\
v(x, 0) & =u_{0}(x), & x & \in \sigma_{h},
\end{aligned}
$$

where $\Delta_{h} v=v_{\bar{x}_{1} x_{1}}+v_{\bar{x}_{2} x_{2}}$ is the difference Laplace operator,

$$
\delta_{\sigma_{h}}(x)=\delta_{h_{2}}\left(x_{2}-x_{2}^{0}\right)= \begin{cases}0, & x \in \omega_{h} \backslash \sigma_{h}, \\ 1 / h_{2}, & x \in \sigma_{h}\end{cases}
$$

is the difference Dirac delta function, and $T_{i}, T_{t}^{-}$are the Steklov averaging operators [15]:

$$
\begin{aligned}
& T_{1} f\left(x_{1}, x_{2}, t\right)=T_{1}^{-} f\left(x_{1}+h_{1} / 2, x_{2}, t\right)=T_{1}^{+} f\left(x_{1}-h_{1} / 2, x_{2}, t\right)=\frac{1}{h_{1}} \int_{x_{1}-h_{1} / 2}^{x_{1}+h_{1} / 2} f\left(x_{1}^{\prime}, x_{2}, t\right) d x_{1}^{\prime}, \\
& T_{2} f\left(x_{1}, x_{2}, t\right)=T_{2}^{-} f\left(x_{1}, x_{2}+h_{2} / 2, t\right)=T_{2}^{+} f\left(x_{1}, x_{2}-h_{2} / 2, t\right)=\frac{1}{h_{2}} \int_{x_{2}-h_{2} / 2}^{x_{2}+h_{2} / 2} f\left(x_{1}, x_{2}^{\prime}, t\right) d x_{2}^{\prime}, \\
& T_{t}^{-} f\left(x_{1}, x_{2}, t\right)=T_{t}^{+} f\left(x_{1}, x_{2}, t-\tau\right)=\frac{1}{\tau} \int_{t-\tau}^{t} f\left(x_{1}, x_{2}, t^{\prime}\right) d t^{\prime} .
\end{aligned}
$$


Note that these operators are self-comutative and transform the derivatives to difference relations, for example,

$$
T_{i}^{-} \frac{\partial u}{\partial x_{i}}=u_{\bar{x}_{i}}, \quad T_{i}^{2} \frac{\partial^{2} u}{\partial x_{i}^{2}}=u_{x_{i} \bar{x}_{i}}, \quad T_{t}^{-} \frac{\partial u}{\partial t}=u_{\bar{t}} .
$$

Let us consider the operator-difference scheme of the form

$$
\begin{aligned}
B_{h} v_{\bar{t}}+A_{h} v & =\varphi(t), \quad t \in \omega_{\tau}^{+}, \\
B_{h} v(0) & =B_{h} v_{0},
\end{aligned}
$$

which corresponds to problem (8). Here $v_{0}$ is a given element in the finite-dimensional Hilbert space $H_{h}, \varphi(t)$ is a given function and $v(t)$ is the unknown function with values in $H_{h}, A_{h}$ is linear operator in $H_{h}, B_{h}$ is a non-negative self-adjoint linear operator in $H_{h}$, and $A_{h} \geqslant B_{h}$. The inner product and the norm in $H_{h}$ are denoted by $(v, w)_{h}$ and $\|v\|_{h}$.

The following analog of Lemma 1 holds [12]:

Lemma 3. For the solution of the problem (10) the following a priori estimates hold

$$
\begin{aligned}
& \tau \sum_{t \in \omega_{\tau}^{+}}\|v(t)\|_{A_{h}}^{2}+\tau^{2} \sum_{t \in \bar{\omega}_{\tau}} \sum_{t^{\prime} \in \bar{\omega}_{\tau}, t^{\prime} \neq t} \frac{\left\|v(t)-v\left(t^{\prime}\right)\right\|_{B_{h}}^{2}}{\left|t-t^{\prime}\right|^{2}}+\max _{t \in \omega_{\tau}^{+}}\|v(t)\|_{B_{h}}^{2} \\
& \leqslant C\left(\left\|v_{0}\right\|_{B_{h}}^{2}+\tau\left\|v_{0}\right\|_{A_{h}}^{2}+\tau \sum_{t \in \omega_{\tau}^{+}}\|\varphi(t)\|_{A_{h}^{-1}}^{2}\right), \\
& \tau \sum_{t \in \omega_{\tau}^{+}}\|v(t)\|_{B_{h}}^{2}+\max _{t \in \omega_{\tau}^{+}}\left\|B_{h} v(t)\right\|_{A_{h}^{-1}}^{2} \leqslant C\left(\left\|B_{h} v_{0}\right\|_{A_{h}^{-1}}^{2}+\tau\left\|v_{0}\right\|_{B_{h}}^{2}+\tau \sum_{t \in \omega_{\tau}^{+}}\left\|A_{h}^{-1} \varphi(t)\right\|_{B_{h}}^{2}\right) .
\end{aligned}
$$

In the case of $\varphi=B_{h} \psi_{\bar{t}}$ the following a priori estimates hold

$$
\begin{gathered}
\tau \sum_{t \in \omega_{\tau}^{+}}\|v(t)\|_{A_{h}}^{2}+\tau^{2} \sum_{t \in \bar{\omega}_{\tau}} \sum_{t^{\prime} \in \bar{\omega}_{\tau}, t^{\prime} \neq t} \frac{\left\|v(t)-v\left(t^{\prime}\right)\right\|_{B_{h}}^{2}}{\left|t-t^{\prime}\right|^{2}}+\max _{t \in \omega_{\tau}^{+}}\|v(t)\|_{B_{h}}^{2} \\
\leqslant C\left\{\left\|v_{0}\right\|_{B_{h}}^{2}+\tau\left\|v_{0}\right\|_{A_{h}}^{2}+\tau^{2} \sum_{t \in \bar{\omega}_{\tau}} \sum_{t^{\prime} \in \bar{\omega}_{\tau}, t^{\prime} \neq t} \frac{\left\|\psi(t)-\psi\left(t^{\prime}\right)\right\|_{B_{h}}^{2}}{\left|t-t^{\prime}\right|^{2}}+\tau \sum_{t \in \omega_{\tau}}\left(\frac{1}{t}+\frac{1}{T-t}\right)\|\psi(t)\|_{B_{h}}^{2}\right\}, \\
\tau \sum_{t \in \omega_{\tau}^{+}}\|v(t)\|_{B_{h}}^{2}+\max _{t \in \omega_{\tau}^{+}}\left\|B_{h} v(t)\right\|_{A_{h}^{-1}}^{2} \leqslant C\left(\left\|B_{h} v_{0}\right\|_{A_{h}^{-1}}^{2}+\max _{t \in \bar{\omega}_{\tau}}\left\|B_{h} \psi(t)\right\|_{A_{h}^{-1}}^{2}+\tau \sum_{t \in \bar{\omega}_{\tau}^{+}}\|\psi(t)\|_{B_{h}}^{2}\right) .
\end{gathered}
$$

Let $H_{h}$ be the space of mesh functions defined on $\omega_{h}$, equal to zero on $\gamma_{h}$, endowed with the inner product and the norm

$$
(v, w)_{h}=h_{1} h_{2} \sum_{x \in \omega_{h}} v(x) w(x), \quad\|v\|_{h}=\|v\|_{L_{2}\left(\omega_{h}\right)}=(v, v)_{h}^{1 / 2} .
$$

We also denote

$$
\begin{gathered}
(v, w)_{i, h}=h_{1} h_{2} \sum_{x_{i} \in \omega_{i}^{-}, x_{3-i} \in \omega_{3-i}} v(x) w(x), \quad\|v\|_{i, h}=(v, v)_{i, h}^{1 / 2}, \\
\|v\|_{W_{2}^{1}\left(\omega_{h}\right)}^{2}=\left\|v_{x_{1}}\right\|_{1, h}^{2}+\left\|v_{x_{2}}\right\|_{2, h}^{2}+\|v\|_{h}^{2}
\end{gathered}
$$


and

$$
(v, w)_{\sigma_{h}}=h_{1} \sum_{x \in \sigma_{h}} v(x) w(x), \quad\|v\|_{\sigma_{h}}=\|v\|_{L_{2}\left(\sigma_{h}\right)}=(v, v)_{\sigma_{h}}^{1 / 2} .
$$

Problem (8) can be reduced to form (10) if one sets $A_{h} v=-\Delta_{h} v$ and $B_{h} v=\delta_{\sigma_{h}} v$. Then

$$
\begin{aligned}
& \|v\|_{A_{h}}^{2}=\left\|v_{x_{1}}\right\|_{1, h}^{2}+\left\|v_{x_{2}}\right\|_{2, h}^{2} \asymp\|v\|_{W_{2}^{1}\left(\omega_{h}\right)}^{2}, \quad v \in H_{h}, \\
& \|v\|_{B_{h}}=\|v\|_{L_{2}\left(\sigma_{h}\right)} .
\end{aligned}
$$

Further we shall make use of the assertion:

Lemma 4. Let $v \in H_{h}$ and $w$ be a mesh function defined on $\sigma_{h}^{-}$. Then

$$
\left|\left(w_{\bar{x}_{1}}, v\right)_{\sigma_{h}}\right| \leqslant C\|v\|_{W_{2}^{1}\left(\omega_{h}\right)}\left\{h_{1}^{2} \sum_{x \in \sigma_{h}^{-}} \sum_{x^{\prime} \in \sigma_{h}^{-}, x^{\prime} \neq x} \frac{\left|w(x)-w\left(x^{\prime}\right)\right|^{2}}{\left|x_{1}-x_{1}^{\prime}\right|^{2}}\right\}^{1 / 2} .
$$

The proof is analogous to the proof of Lemma 2 in [10].

\section{Convergence of the difference approximation}

Let $u$ be the solution of the IBVP (2) and $v$ - the solution of the difference scheme (8). The error $z=u-v$ satisfies the difference problem

$$
\begin{aligned}
\delta_{\sigma_{h}} z_{\bar{t}}-\Delta_{h} z & =\phi, & (x, t) & \in \omega_{h} \times \omega_{\tau}^{+}, \\
z(x, t) & =0, & (x, t) & \in \gamma_{h} \times \omega_{\tau}^{+}, \\
z(x, 0) & =0, & x & \in \sigma_{h},
\end{aligned}
$$

where

$$
\phi=\delta_{\sigma_{h}} \xi_{\bar{t}}+\eta_{1, \bar{x}_{1} x_{1}}+\eta_{2, \bar{x}_{2} x_{2}}, \quad \xi=u-T_{1}^{2} u, \quad \eta_{i}=T_{3-i}^{2} T_{t}^{-} u-u .
$$

Let us set

$$
\zeta=\frac{h_{2}^{2}}{6}\left[T_{t}^{-} \frac{\partial u}{\partial x_{2}}\right]_{S}, \quad x \in \sigma_{h} ; \quad \widetilde{\eta}_{1}=\eta_{1}-\delta_{\sigma_{h}} \zeta .
$$

We rewrite the difference scheme (11) as the operator difference scheme

$$
\begin{aligned}
B_{h} z_{\bar{t}}+A_{h} z & =\phi(t), \quad t \in \omega_{\tau}^{+}, \\
B_{h} z(0) & =0,
\end{aligned}
$$

where $A_{h} z=-\Delta_{h} z, \quad B_{h} z=\delta_{\sigma_{h}} z, \quad \phi=\delta_{\sigma_{h}} \xi_{\bar{t}}+\widetilde{\eta}_{1, \bar{x}_{1} x_{1}}+\eta_{2, \bar{x}_{2} x_{2}}+\delta_{\sigma_{h}} \zeta_{\bar{x}_{1} x_{1}}$.

Using Lemmas 3 and 4 , we get the a priori estimate

$$
\begin{gathered}
\tau \sum_{t \in \omega_{\tau}^{+}}\|z(\cdot, t)\|_{W_{2}^{1}\left(\omega_{h}\right)}^{2}+\tau^{2} \sum_{t \in \bar{\omega}_{\tau}} \sum_{t^{\prime} \in \bar{\omega}_{\tau}, t^{\prime} \neq t} \frac{\left\|z(\cdot, t)-z\left(\cdot, t^{\prime}\right)\right\|_{L_{2}\left(\sigma_{h}\right)}^{2}}{\left|t-t^{\prime}\right|^{2}}+\max _{t \in \omega_{\tau}^{+}}\|z(\cdot, t)\|_{L_{2}\left(\sigma_{h}\right)}^{2} \\
\leqslant C\left\{\tau \sum_{t \in \omega_{\tau}^{+}}\left(\left\|\widetilde{\eta}_{1, x_{1}}(\cdot, t)\right\|_{1, h}^{2}+\left\|\eta_{2, x_{2}}(\cdot, t)\right\|_{2, h}^{2}\right)\right. \\
+\tau \sum_{t \in \omega_{\tau}^{+}} h_{1}^{2} \sum_{x \in \sigma_{h}^{-}} \sum_{x^{\prime} \in \sigma_{h}^{-}, x^{\prime} \neq x} \frac{\left|\zeta_{x_{1}}(x, t)-\zeta_{x_{1}}\left(x^{\prime}, t\right)\right|^{2}}{\left|x_{1}-x_{1}^{\prime}\right|^{2}} \\
+\tau^{2} \sum_{t \in \bar{\omega}_{\tau}} \sum_{\left.t_{t^{\prime} \in \bar{\omega}_{\tau}, t^{\prime} \neq t} \frac{\left\|\xi(\cdot, t)-\xi\left(\cdot, t^{\prime}\right)\right\|_{L_{2}\left(\sigma_{h}\right)}^{2}}{\left|t-t^{\prime}\right|^{2}}+\tau \sum_{t \in \omega_{\tau}}\left(\frac{1}{t}+\frac{1}{T-t}\right)\|\xi(\cdot, t)\|_{L_{2}\left(\sigma_{h}\right)}^{2}\right\} .}
\end{gathered}
$$


Therefore, in order to estimate the rate of convergence of the difference scheme (8), it is sufficient to estimate the norms of $\xi, \widetilde{\eta}_{1}, \eta_{2}$ and $\zeta$ on the right-hand side of (12).

We begin with $\eta_{2}$. Letting

$$
\eta_{2}=\eta_{21}+\eta_{22}+\eta_{23}=\left(T_{1}^{2} T_{t}^{-} u-T_{t}^{-} u\right)+\left(T_{1}^{2} T_{t}^{-} u-T_{1}^{2} u\right)+\left(T_{t}^{-} u-u-T_{1}^{2} T_{t}^{-} u+T_{1}^{2} u\right)
$$

and using (9), it is easy to find the integral representations

$$
\eta_{21, x_{2}}=\frac{1}{h_{1} h_{2} \tau} \int_{x_{1}-h_{1}}^{x_{1}+h_{1}} \int_{x_{1}}^{x_{1}} \int_{x_{2}}^{x_{1}^{\prime}} \int_{t-\tau}^{x_{1}^{\prime \prime}}\left(1-\frac{\left|x_{1}^{\prime}-x_{1}\right|}{h_{1}}\right) \frac{\partial^{3} u}{\partial x_{1}^{2} \partial x_{2}}\left(x_{1}^{\prime \prime \prime}, x_{2}^{\prime}, t^{\prime}\right) d t^{\prime} d x_{2}^{\prime} d x_{1}^{\prime \prime \prime} d x_{1}^{\prime \prime} d x_{1}^{\prime}
$$

and

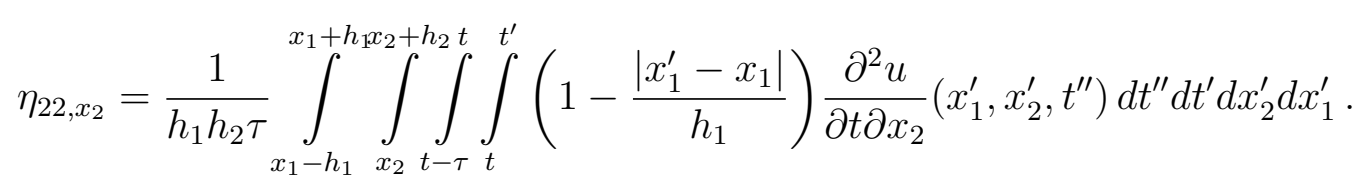

Hence

$$
\left(\tau \sum_{t \in \omega_{\tau}^{+}}\left\|\eta_{21, x_{2}}\right\|_{2, h}^{2}\right)^{1 / 2} \leqslant C h_{1}^{2}\left\|\frac{\partial^{3} u}{\partial x_{1}^{2} \partial x_{2}}\right\|_{L_{2}(Q)}, \quad\left(\tau \sum_{t \in \omega_{\tau}^{+}}\left\|\eta_{22, x_{2}}\right\|_{2, h}^{2}\right)^{1 / 2} \leqslant C \tau\left\|\frac{\partial^{3} u}{\partial t \partial x_{2}}\right\|_{L_{2}(Q)} .
$$

Using the technique from [9], we get

$$
\begin{aligned}
\left(\tau \sum_{t \in \omega_{\tau}^{+}}\left\|\eta_{23, x_{2}}\right\|_{2, h}^{2}\right)^{1 / 2} & \leqslant C\left(h_{1}^{2}+\tau\right)\left(h_{2} \sum_{x_{2} \in \omega_{2}^{-}}\left\|T_{2}^{+} \frac{\partial u}{\partial x_{2}}\left(\cdot, x_{2}, \cdot\right)\right\|_{W_{2}^{2,1}\left(\left(0, l_{1}\right) \times(0, T)\right)}^{2}\right)^{1 / 2} \\
& \leqslant C\left(h_{1}^{2}+\tau\right)\left(\left\|\frac{\partial^{3} u}{\partial x_{1}^{2} \partial x_{2}}\right\|_{L_{2}(Q)}+\left\|\frac{\partial^{2} u}{\partial t \partial x_{2}}\right\|_{L_{2}(Q)}+\left\|\frac{\partial u}{\partial x_{2}}\right\|_{L_{2}(Q)}\right) .
\end{aligned}
$$

The last three estimates give

$$
\left(\tau \sum_{t \in \omega_{\tau}^{+}}\left\|\eta_{2, x_{2}}\right\|_{2, h}^{2}\right)^{1 / 2} \leqslant C\left(h_{1}^{2}+\tau\right)\left(\left\|\frac{\partial^{3} u}{\partial x_{1}^{2} \partial x_{2}}\right\|_{L_{2}(Q)}+\left\|\frac{\partial^{2} u}{\partial t \partial x_{2}}\right\|_{L_{2}(Q)}+\left\|\frac{\partial u}{\partial x_{2}}\right\|_{L_{2}(Q)}\right) .
$$

In a similar way, we let $\widetilde{\eta}_{1}=\widetilde{\eta}_{11}+\widetilde{\eta}_{12}+\widetilde{\eta}_{13}$, where

$$
\begin{aligned}
& \widetilde{\eta}_{11}=\left\{\begin{array}{ll}
T_{2}^{2} T_{t}^{-} u-T_{t}^{-} u, & x \in \omega_{h} \backslash \sigma_{h}, \\
0.5\left(\widetilde{\eta}_{11}^{+}+\widetilde{\eta}_{11}^{-}\right), & x \in \sigma_{h},
\end{array} \widetilde{\eta}_{11}^{ \pm}=T_{2 \pm}^{2} T_{t}^{-} u-\left.T_{t}^{-} u \mp \frac{h_{2}}{3} T_{t}^{-} \frac{\partial u}{\partial x_{2}}\right|_{x_{2}=x_{2}^{0} \pm 0},\right. \\
& \widetilde{\eta}_{12}=T_{2}^{2} T_{t}^{-} u-T_{2}^{2} u, \quad x \in \omega_{h}, \\
& \widetilde{\eta}_{13}=\left\{\begin{array}{ll}
T_{t}^{-} u-u-T_{2}^{2} T_{t}^{-} u+T_{2}^{2} u, & x \in \omega_{h} \backslash \sigma_{h}, \\
0.5\left(\widetilde{\eta}_{13}^{+}+\widetilde{\eta}_{13}^{-}\right), & x \in \sigma_{h},
\end{array} \widetilde{\eta}_{13}^{ \pm}=T_{t}^{-} u-u-T_{2 \pm}^{2} T_{t}^{-} u+T_{2 \pm}^{2} u\right.
\end{aligned}
$$

and

$$
T_{2-}^{2} u=\frac{2}{h_{2}} \int_{x_{2}^{0}-h_{2}}^{x_{2}^{0}}\left(1+\frac{x_{2}^{\prime}-x_{2}^{0}}{h_{2}}\right) u\left(x_{1}, x_{2}^{\prime}, t\right) d x_{2}^{\prime}, \quad T_{2+}^{2} u=\frac{2}{h_{2}} \int_{x_{2}^{0}}^{x_{2}^{0}+h_{2}}\left(1-\frac{x_{2}^{\prime}-x_{2}^{0}}{h_{2}}\right) u\left(x_{1}, x_{2}^{\prime}, t\right) d x_{2}^{\prime} .
$$


When $x \in \omega_{h} \backslash \sigma_{h}$ the expressions $\widetilde{\eta}_{1 j}$ can be estimated in a similar way as their corresponding $\eta_{2 j}(j=1,2,3)$. When $x \in \sigma_{h}$ the expression $\widetilde{\eta}_{13}^{ \pm}$can be estimated as $\widetilde{\eta}_{13}$ when $x \in \omega_{h} \backslash \sigma_{h}$. Further,

$$
\widetilde{\eta}_{11, x_{1}}^{+}=\frac{1}{h_{1} h_{2} \tau} \int_{x_{1}}^{x_{1}+h_{1} x_{2}^{0}} \int_{x_{2}^{0}} \int_{x_{2}^{0}}^{+h_{2} x_{2}^{\prime}} \int_{x_{2}^{0}}^{x_{2}^{\prime \prime}} \int_{t-\tau}^{t}\left(1-\frac{x_{2}^{\prime}-x_{2}}{h_{2}}\right) \frac{\partial^{3} u}{\partial x_{1} \partial x_{2}^{2}}\left(x_{1}^{\prime}, x_{2}^{\prime \prime \prime}, t^{\prime}\right) d t^{\prime} d x_{2}^{\prime \prime \prime} d x_{2}^{\prime \prime} d x_{2}^{\prime} d x_{1}^{\prime}
$$

and an alogous representation holds for $\widetilde{\eta}_{11, x_{1}}^{-}$. Reasoning as in the last case, we get the estimate

$$
\begin{aligned}
\left(\tau \sum_{t \in \omega_{\tau}^{+}}\left\|\widetilde{\eta}_{1, x_{1}}\right\|_{2}^{2}\right)^{1 / 2} \leqslant & C\left(h_{2}^{2}+\tau\right)\left(\left\|\frac{\partial^{3} u}{\partial x_{2}^{2} \partial x_{1}}\right\|_{L_{2}\left(Q^{-}\right)}+\left\|\frac{\partial^{3} u}{\partial x_{2}^{2} \partial x_{1}}\right\|_{L_{2}\left(Q^{+}\right)}\right. \\
& \left.+\left\|\frac{\partial^{2} u}{\partial t \partial x_{1}}\right\|_{L_{2}(Q)}+\left\|\frac{\partial u}{\partial x_{1}}\right\|_{L_{2}(Q)}\right) .
\end{aligned}
$$

The integral representation

$$
\zeta_{x_{1}}=\frac{h_{2}^{2}}{6 h_{1} \tau} \int_{t-\tau}^{t} \int_{x_{1}}^{x_{1}+h_{1}}\left(\frac{\partial^{2} u}{\partial x_{1} \partial x_{2}}\left(x_{1}^{\prime \prime}, x_{2}^{0}+0, t\right)-\frac{\partial^{2} u}{\partial x_{1} \partial x_{2}}\left(x_{1}^{\prime \prime}, x_{2}^{0}-0, t\right)\right) d x_{1}^{\prime \prime} d t^{\prime}
$$

and the imbedding theorem imply

$$
\begin{aligned}
& \left.\left\{\tau \sum_{t \in \omega_{\tau}^{+}} h_{1}^{2} \sum_{x \in \sigma_{h}^{-}} \sum_{x^{\prime} \in \sigma_{h}^{-}, x^{\prime} \neq x} \frac{\left|\zeta_{x_{1}}(x, t)-\zeta_{x_{1}}\left(x^{\prime}, t\right)\right|^{2}}{\left|x_{1}-x_{1}^{\prime}\right|^{2}}\right\}^{1 / 2} \leqslant \frac{h_{2}^{2}}{6} \sqrt{\frac{7}{6}}\left\|\left[\frac{\partial^{2} u}{\partial x_{1} \partial x_{2}}\right]_{S}\right\|_{L_{2}\left(0, T ; W_{2}^{1 / 2}(S)\right)}\right) \\
& \quad \leqslant C h_{2}^{2}\left(\left\|\frac{\partial^{2} u}{\partial x_{1} \partial x_{2}}\right\|_{L_{2}\left(0, T ; W_{2}^{1}\left(\Omega^{-}\right)\right)}+\left\|\frac{\partial^{2} u}{\partial x_{1} \partial x_{2}}\right\|_{L_{2}\left(0, T ; W_{2}^{1}\left(\Omega^{+}\right)\right)}\right) \\
& \quad \leqslant C h_{2}^{2}\left(\left\|\frac{\partial^{3} u}{\partial x_{1}^{2} \partial x_{2}}\right\|_{L_{2}(Q)}+\left\|\frac{\partial^{3} u}{\partial x_{1} \partial x_{2}^{2}}\right\|_{L_{2}\left(Q^{-}\right)}+\left\|\frac{\partial^{3} u}{\partial x_{1} \partial x_{2}^{2}}\right\|_{L_{2}\left(Q^{+}\right)}+\left\|\frac{\partial^{2} u}{\partial x_{1} \partial x_{2}}\right\|_{L_{2}(Q)}\right) .
\end{aligned}
$$

The terms containing the expression $\xi$ can be estimated as in [12]. Therefore, we get

$$
\left\{\tau^{2} \sum_{t \in \bar{\omega}_{\tau}} \sum_{t^{\prime} \in \bar{\omega}_{\tau}, t^{\prime} \neq t} \frac{\left\|\xi(\cdot, t)-\xi\left(\cdot, t^{\prime}\right)\right\|_{L_{2}\left(\sigma_{h}\right)}^{2}}{\left|t-t^{\prime}\right|^{2}}\right\}^{1 / 2} \leqslant C\left(h_{1}^{2}+\tau\right)\|u\|_{W_{2}^{3,3 / 2}(S \times(0, T))}
$$

and

$$
\left\{\tau \sum_{t \in \omega_{\tau}}\left(\frac{1}{t}+\frac{1}{T-t}\right)\|\xi(\cdot, t)\|_{L_{2}\left(\sigma_{h}\right)}^{2}\right\}^{1 / 2} \leqslant C h_{1}^{2} \sqrt{\ln 1 / \tau}\|u\|_{W_{2}^{3,3 / 2}(S \times(0, T))} .
$$

Now, from (12)-(17) we obtain the desired estimate for the convergence rate of the difference scheme (8):

$$
\begin{aligned}
& \left\{\tau \sum_{t \in \omega_{\tau}^{+}}\|z(\cdot, t)\|_{W_{2}^{1}\left(\omega_{h}\right)}^{2}+\tau^{2} \sum_{t \in \bar{\omega}_{\tau}} \sum_{t^{\prime} \in \bar{\omega}_{\tau}, t^{\prime} \neq t} \frac{\left\|z(\cdot, t)-z\left(\cdot, t^{\prime}\right)\right\|_{L_{2}\left(\sigma_{h}\right)}^{2}}{\left|t-t^{\prime}\right|^{2}}+\max _{t \in \omega_{\tau}^{+}}\|z(\cdot, t)\|_{L_{2}\left(\sigma_{h}\right)}^{2}\right\}^{1 / 2} \\
& \leqslant C\left(h_{1}^{2}+h_{2}^{2}+\tau\right) \sqrt{\ln 1 / \tau}\left(\left\|\frac{\partial^{3} u}{\partial x_{1}^{2} \partial x_{2}}\right\|_{L_{2}(Q)}+\left\|\frac{\partial^{3} u}{\partial x_{2}^{2} \partial x_{1}}\right\|_{L_{2}\left(Q^{-}\right)}+\left\|\frac{\partial^{3} u}{\partial x_{2}^{2} \partial x_{1}}\right\|_{L_{2}\left(Q^{+}\right)}\right. \\
& \left.\quad+\left\|\frac{\partial^{2} u}{\partial t \partial x_{1}}\right\|_{L_{2}(Q)}+\left\|\frac{\partial^{2} u}{\partial t \partial x_{2}}\right\|_{L_{2}(Q)}+\left\|\frac{\partial u}{\partial x_{1}}\right\|_{L_{2}(Q)}+\left\|\frac{\partial u}{\partial x_{2}}\right\|_{L_{2}(Q)}+\|u\|_{W_{2}^{3,3 / 2}(S \times(0, T))}\right) .
\end{aligned}
$$


Thus, in view of Lemma 2, we have proved the main result of the paper.

Theorem 1. Let $z=u-v$ be the error and the conditions of Lemma 2 are fulfilled. Then the difference scheme (8) converges and the following estimate holds:

$$
\begin{aligned}
& \quad\left\{\tau \sum_{t \in \omega_{\tau}^{+}}\|z(\cdot, t)\|_{W_{2}^{1}\left(\omega_{h}\right)}^{2}+\tau^{2} \sum_{t \in \bar{\omega}_{\tau}} \sum_{t^{\prime} \in \bar{\omega}_{\tau}, t^{\prime} \neq t} \frac{\left\|z(\cdot, t)-z\left(\cdot, t^{\prime}\right)\right\|_{L_{2}\left(\sigma_{h}\right)}^{2}}{\left|t-t^{\prime}\right|^{2}}+\max _{t \in \omega_{\tau}^{+}}\|z(\cdot, t)\|_{L_{2}\left(\sigma_{h}\right)}^{2}\right\}^{1 / 2} \\
& \leqslant C\left(h_{1}^{2}+h_{2}^{2}+\tau\right) \sqrt{\ln 1 / \tau}\left(\left\|u_{0}\right\|_{W_{2}^{2}(S)}+\|f(\cdot, 0)\|_{L_{2}(\Omega)}+\|f\|_{L_{2}\left(0, T ; W_{2}^{1}(\Omega)\right)}\right. \\
& \left.\quad+\|f\|_{W_{2}^{1}\left(0, T ; W_{2}^{-1}(\Omega)\right)}+\|g\|_{L_{2}\left(0, T ; W_{2}^{3 / 2}(S)\right)}+\|g\|_{\widetilde{W}_{2}^{1 / 2}\left(0, T ; L_{2}(S)\right)}\right) .
\end{aligned}
$$

Remark 2. An analogous result holds for the weighted scheme

$$
\begin{aligned}
& \delta_{\sigma_{h}} v_{\bar{t}}-\Delta_{h} v^{(\kappa)}=T_{1}^{2} T_{2}^{2} T_{t}^{-} \varphi, \quad(x, t) \in \omega_{h} \times \omega_{\tau}^{+}, \\
& v(x, t)=0, \quad(x, t) \in \gamma_{h} \times \omega_{\tau}^{+}, \\
& v(x, 0)=u_{0}(x), \quad x \in \sigma_{h},
\end{aligned}
$$

where $v^{(\kappa)}=\kappa v(x, t)+(1-\kappa) v(x, t-\tau)$. The condition $\kappa \geqslant 1 / 2$ is assumed.

Remark 3. In the case of a lower smoothness of the input data $\left(u_{0} \in \stackrel{\circ}{W_{2}^{1}}(S), f \in L_{2}(Q)\right.$, $\left.g \in L_{2}\left(0, T ; \widetilde{W}_{2}^{1 / 2}(S)\right)\right)$, one can prove the convergence of the difference scheme (8) in the weaker norm

$$
\left\{\tau \sum_{t \in \omega_{\tau}^{+}}\|z(t)\|_{B_{h}}^{2}+\max _{t \in \omega_{\tau}^{+}}\left\|B_{h} z(t)\right\|_{A_{h}^{-1}}^{2}\right\}^{1 / 2} .
$$

Acknowledgement: The research of the first author was supported by the MSTD of the Republic of Serbia under grant 1840 and the research of the second author was supported by the University of Rousse under grant 2002-PF-04.

\section{References}

[1] V.B. Andreev, Stability of difference schemes for elliptic equations with Dirichlet's boundary conditions, Zh. Vychisl. Mat. Mat. Fiz., 12 (1972), pp. 598-611 (in Russian).

[2] J.H. Bramble and S.R. Hilbert, Bounds for a class of linear functionals with application to Hermite interpolation, Numer. Math., 16 (1971), pp. 362-369.

[3] I. A. Brayanov and L. G. Vulkov, Homogeneous difference schemes for the heat equation with concentrated capacity, Zh. Vychisl. Mat. Mat. Fiz., 39 (1999), pp. 254-261 (in Russian).

[4] R. W. Caroll and R.E. Showalter, Singular and degenerate Cauchy problems, Mathematics in Science and Engineering, Vol. 127, Academic Press, New York, San Francisco, London, 1976.

[5] T. Dupont and R. Scott, Polynomial approximation of functions in Sobolev spaces, Math. Comput., 34 (1980), pp. 441-463.

[6] J. Escher, Quasilinear parabolic systems with dynamical boundary conditions, Communs Partial Differential Equations, 19 (1993), pp. 1309-1364.

[7] B.S. Jovanović, Finite difference method for boundary value problems with weak solutions, Posebna izdanja Mat. Instituta, 16, Belgrade, 1993. 
[8] B.S. Jovanović and P.P. Matus, Strong stability of operator-differential equations and operatordifference schemes in norms integral with respect to time. Differencial'nye uravneniya, 37 (2001), pp. 950-958 (in Russian).

[9] B.S. Jovanović, P.P. Matus, and V.S. Shcheglik, Finite difference schemes on nonuniform meshes for parabolic equation with variable coefficients and generalized solutions, Doklady NAN Belarusi 42 (1998), No. 6, pp. 38-44 (in Russian).

[10] B.S. Jovanović and B.Z. Popović, Convergence of a finite difference scheme for the third boundaryvalue problem for an elliptic equation with variable coefficients, Comp. Methods Appl. Math., 1 (2001), No. 1, pp. 356-366.

[11] B. S. Jovanović and L. G. Vulkov, Operator's approach to the problems with concentrated factors, Lect. Notes Comput. Sci., 1988 (2001), pp. 439-450.

[12] B.S. Jovanović and L. G. Vulkov, On the convergence of finite difference schemes for the heat equation with concentrated capacity, Numer. Math., 89 (2001), No. 4, pp. 715-734.

[13] B. S. Jovanović and L. G. Vulkov, On the convergence of finite difference schemes for hyperbolic equations with concentrated factors, SIAM J. Numer. Analysis (accepted).

[14] O. A. Ladyzhenskaya, V. A. Solonnikov, and N. N. Ural'tseva, Linear and Quasilinear Equations of Parabolic Type, Nauka, Moscow, 1967 (in Russian).

[15] R. D. Lazarov, V.L. Makarov, and A. A. Samarskiǔ, Applications of exact difference schemes for construction and studies of difference schemes on generalized solutions, Math. Sbornik, 117 (1982), pp. 469-480 (in Russian).

[16] A. V. Lykov, Heat-Mass Transfer, Energiya, Moscow, 1978 (in Russian).

[17] J. L. Lions and E. Magenes, Non Homogeneous Boundary Value Problems and Applications, SpringerVerlag, Berlin and New York, 1972.

[18] A. G. Rutkas, Cauchy problem for the equation $A x^{\prime}(t)+B x(t)=f(t)$, Differentsial'nye Uravneniya, 11 (1975), pp. 1996-2010 (in Russian).

[19] A. A. Samarskiu,, Theory of Difference Schemes, Nauka, Moscow, 1989 (in Russian).

[20] A. A. Samarskiŭ, R. D. Lazarov, and V. L. Makarov, Difference Schemes for Differential Equations with Generalized Solutions, Vyshaya Shkola, Moscow, 1987 (in Russian).

[21] P. N. Vabishchevich, Numerical solution of an elliptic problem with nonstationary boundary conditions, Mat. Modelirovanie, 7 (1995), No. 7, pp. 49-60 (in Russian).

[22] V.S. Vladimirov, Equations of Mathematical Physics, Nauka, Moscow, 1988 (in Russian).

[23] L. Vulkov, Application of Steklov-type eigenvalues problems to convergence of difference schemes for parabolic and hyperbolic equation with dynamical boundary conditions, Lect. Notes Comput. Sci., 1196 (1997), pp. 557-564.

[24] J. Wloka, Partial Differential Equations, Cambridge Univ. Press, Cambridge, 1987.

Received 10 Jan. 2003

Revised 24 Feb. 2003 